\title{
How the Covid-19 epidemic is challenging our practice in clinical nutrition-feedback from the field
}

\author{
Ronan Thibault $\mathbb{1}^{1} \cdot$ Moïse Coëffier ${ }^{2} \cdot$ Francisca Joly ${ }^{3}$ Julien Bohé ${ }^{4} \cdot$ Stéphane M. Schneider $\mathbb{C}^{5}$ • \\ Pierre Déchelotte $\mathbb{B}^{2}$
}

Received: 16 June 2020 / Revised: 28 August 2020 / Accepted: 5 September 2020 / Published online: 16 September 2020

(c) The Author(s), under exclusive licence to Springer Nature Limited 2020

\begin{abstract}
The viral epidemic caused by the new Coronavirus SARS-CoV-2 is responsible for the new Coronavirus disease-2019 (Covid-19). Fifteen percent of the Covid-19 patients will require hospital stay, and $10 \%$ of them will need urgent respiratory and hemodynamic support in the intensive care unit (ICU). Covid-19 is an infectious disease characterized by inflammatory syndrome, itself leading to reduced food intake and increased muscle catabolism. Therefore Covid-19 patients are at high risk of being malnourished, making the prevention of malnutrition and the nutritional management key aspects of care. Urgent, brutal and massive arrivals of patients needing urgent respiratory care and artificial ventilation lead to the necessity to reorganize hospital care, wards and staff. In that context, nutritional screening and care may not be considered a priority. Moreover, at the start of the epidemic, due to mask and other protecting material shortage, the risk of healthcare givers contamination have led to not using enteral nutrition, although indicated, because nasogastric tube insertion is an aerosolgenerating procedure. Clinical nutrition practice based on the international guidelines should therefore adapt and the use of degraded procedures could unfortunately be the only way. Based on the experience from the first weeks of the epidemic in France, we emphasize ten challenges for clinical nutrition practice. The objective is to bring objective answers to the most frequently met issues to help the clinical nutrition caregivers to promote nutritional care in the hospitalized Covid-19 patient. We propose a flow chart for optimizing the nutrition management of the Covid-19 patients in the non-ICU wards.
\end{abstract}

\section{General context}

The viral epidemic caused by the new Coronavirus SARS$\mathrm{CoV}-2$ is responsible for the new Coronavirus disease-2019 (Covid-19) [1]. In the Chinese experience, up to $30 \%$ of the

Ronan Thibault

ronan.thibault@chu-rennes.fr

1 Unité de Nutrition, INRAE, INSERM, Nutrition Métabolismes et Cancer, NuMeCan, CHU Rennes, Univ Rennes, Rennes, France

2 Département de Nutrition-CIC1404, CHU Rouen, UNIROUEN, Inserm UMR, Normandie Univ, 1073 Rouen, France

3 Gastroentérologie, MICI, et Assistance Nutritive, AP-HP, Université de Paris, Inserm UMR 1149, F-92110 Clichy, France

4 Service d'anesthésie-Réanimation-Médecine intensive, Centre Hospitalier Lyon-Sud, Hospices Civils de Lyon, Pierre Bénite, France

5 Gastroentérologie et Nutrition, CHU Nice, Université Côte d'Azur, Nice, France
Coronavirus SARS-CoV-2 infected patients are presenting with an acute respiratory distress syndrome (ARDS) requiring urgent respiratory and hemodynamic support in the intensive care unit (ICU) [2]. Covid-19 patients develop in 20\% of cases a severe pneumonia requiring hospitalization. Because of ARDS, the overall mortality rate during Covid-19 infection is estimated to be around 1-2\% [2]. The median intra-hospital mortality rate is around $30 \%$.

The cross-sectional study by $\mathrm{Li}$ et al. performed in hospitalized elderly patients with Covid-19 reported that 53\% of patients were malnourished [3]. This means that nutritional support should be integrated in the global management of the Covid-19 patient. Therefore, we and other members of the French-speaking society for clinical nutrition and metabolism (SFNCM) elaborated practical guidance sheets (www.sfncm.org) to help the French-speaking health caregivers to manage malnutrition and risk for malnutrition in the Covid-19 patients. Our feedback from the field meant we quickly observed that some of the international guidelines of nutritional screening and support (e.g., from ESPEN, ASPEN) were no longer feasible in daily 
practice, because of the Covid-19 epidemic totally challenged our usual routine. Moreover, numerous French hospitals face staff reorganization and sometimes also material shortage. Thus, clinical nutrition practice should adapt, and the use of degraded procedures may unfortunately be the only way.

In the first part of this review article, we will report why Covid-19 patients are at high risk for malnutrition, and propose a rationale to emphasize nutritional management as being a key part of the multidisciplinary care of the Covid19. Based on the experience from the first weeks of the Covid-19 epidemic in France, we then emphasize ten challenges for clinical nutrition practice, and suggest a flow chart for optimizing the nutrition management of the Covid19 patients in the non-ICU wards.

\section{Why is the Covid-19 patient at high risk for malnutrition}

Recent studies or guidelines state that the Covid-19 patient at high risk for malnutrition [3-7]. The most severe cases are encountered in particular, but not exclusively, in patients with a chronic disease (such as organ failure, obesity with body mass index $(\mathrm{BMI}) \geq 35[8,9]$, type 2 diabetes 2 or cancers), elderly and/or polymorbid conditions [10]. These diseases often mask underlying protein malnutrition and sarcopenia [11, 12]. Covid-19 patients should be considered at high risk of malnutrition [3-7], like in other severe respiratory infections characterized by: inflammatory syndrome and hypercatabolism, increased energy expenditure linked to ventilation work $[5,6]$. In the absence of early and adequate nutritional support, this combination will lead to a rapid deterioration of respiratory muscle function, aggravating the consequences of viral pulmonary damage $[4,5]$. Hypermetabolism and physical immobilization expose to rapid muscle wasting because of concomitant drastic reduction in food intake [4-7], which is secondary to several factors: anorexia, dyspnea, dysosmia, and dysgueusia [13]. Finally, digestive symptoms (diarrhea, vomiting, or abdominal pain) [14-17], stress, confinement, organizational problems, and staff shortage will contribute to limit meal offering and ingestion. Importantly, obesity is associated with severe forms of the Covid-19 infections [8,9]. Obesity is also associated with increased protein catabolism and insulin resistance as compared with nonobese patients [18], and preexisting obesity may limit the awareness of nursing staff about providing minimal caloric and increased protein supply to these patients.

Acute malnutrition induced by Covid-19 infection would therefore be associated with increased loss in muscle mass and weakening of immune defenses which together would contribute to the severity of the Covid-19. The malnutrition phenotypic criteria could be a low BMI, a significant weight loss or a low fat-free mass [19]. However, only BMI, already known as very poorly sensitive in diagnosing malnutrition, has been reported in Chinese [20] and NewYork cohort studies [21]; in these studies, low BMI was not predictive of severe forms; this may reflect the limited number of patients in this BMI range and the fact that obese patients displaying acute malnutrition were not detected. Thus, to our opinion, these findings are not eliminating protein energy malnutrition as a potential risk factor for severe Covid-19 forms, as suggested by a more recent study from Asia [22].

\section{Rationale for nutrition support in the Covid-19 patient}

\section{Importance of nutrition support in patients with malnutrition or risk for malnutrition}

General guidelines of The European Society for Clinical Nutrition and Metabolism (ESPEN) recommend prescription of nutritional support for medical, polymorbid in patients at risk of malnutrition [12]. A recent prospective, randomized, unblinded, multicenter trial demonstrated that providing early nutritional support, mostly orally, in hospitalized patients at risk of malnutrition significantly decreased 30-day complications and mortality in comparison to patients on standard hospital diet [23]. The high probability of malnutrition [3-7] and its association with a worse clinical outcome [24] are justifying an active strategy of nutrition screening and support in the Covid-19 patient (https://www.nutritioncare.org/uploadedFiles/Documents/ Guidelines_and_Clinical_Resources/Nutrition\%20Therapy \%20COVID-19_SCCM-ASPEN.pdf) [4, 5, 25]. This would help the patients reinforcing their immune defenses and potentially limiting the evolution toward a severe form of the disease.

\section{Role of nutrition support on immune response}

The innate immune response (i.e., humoral immunity and effector $\mathrm{T}$ cells) appears to be impaired in the Covid-19 patients [2], and the "cured" patients showed better restoration of the number and function of natural killer lymphocytes and CD8 $+\mathrm{T}$ lymphocytes [26]. In severe Covid-19 patients, lymphopenia is common; lymphopenia, innate immune response, and antioxidant defenses are also independently worsened by malnutrition, leading to increased risk of complications and ARDS.

Provision of sufficient protein intake is critical during acute infection and malnutrition. Amino acids, and in particular glutamine, are essential energy substrates for immune cells such as lymphocytes [27]. Activation of 
immune cells requires an increase in the intracellular influx of amino acids and an increase in membrane transporters of amino acids [28]. In addition, the intracellular content of glutathione, a tripeptide with significant antioxidant properties, is a key element in regulating the number and function of lymphocytes [29]. A short fast induces a reduction in the intracellular glutathione content [30], which is also observed in malnourished patients [31, 32].

Caloric restriction also quickly leads to lymphopenia and atrophy of the lymphoid organs [33, 34]. Malnutritionassociated lymphopenia partly explains the increased risk of infectious complications [35]. Therefore, fasting and malnutrition have a negative impact on the immune response.

\section{Expected benefits of nutrition support in the Covid-19 patients}

Early nutritional support could limit these negative consequences [34, 36]. These beneficial effects of refeeding could be due to different mechanisms, including amino acids supply, restoration of the intracellular glutathione content and limitation of protein breakdown.

Furthermore, gastrointestinal (GI) symptoms [14-17] can increase the risk of malnutrition and micronutrient deficiencies. Two Chinese case series from 651 to 1141 Covid19 patients estimated the prevalence of GI symptoms (nausea, diarrhea, and vomiting) from $11.4 \%$ to $16 \%$, respectively [37, 38]. In an analysis of data from the Hong Kong cohort of patients and a meta-analysis, authors found that $17.6 \%$ of Covid-19 patients had GI symptoms and virus RNA fragments were detected in stool samples from $48.1 \%$ patient, even in stools collected after respiratory samples became negative [15].

In that context of GI Covid-19, it is expected that gut microbiota could be modified and that this could worsen the Covid-19 lung damage. Indeed, rapid and severe changes in the gut microbiota [39], a decrease in gut barrier function and increased intestinal permeability [40] could promote changes in the lung microbiota and worsen lung damage [41] leading to ARDS [42]. Conversely, the protective effect induced by a modification of the intestinal microbiota during a viral infection such as the influenza involved a stimulation of the innate immune response [43]. By stimulating the innate immune response and modifying gut microbiota toward a protective microbiota, early enteral nutrition could provide benefit to Covid-19 patients. Indeed, experimental studies have shown favorable changes in the gut microbiota, restoration of the gut barrier function, and stimulation of the innate immune response in animals subjected to EN [44, 45].

Altogether, these data suggest that, in patients infected with the SARS-Cov-2 virus, insufficient nutritional intake could lead to rapid degradation of the intestinal barrier function, of muscle functional capacities, and of immune response, which could thus favor secondary onset of signs of severity and progression to ARDS. To face this increased morbidity related to early and rapid malnutrition, intensive oral or enteral nutrition support should be considered. Indeed, a recent meta-analysis showed that early EN reduced the mortality rate and complications, particularly pneumonia, in critically ill patients compared to EN introduced later after insufficient oral refeeding [46]. The mechanisms explaining this benefit of early EN are undoubtedly multiple.

Although the risk of malnutrition due to severe hypoxia and high-grade systemic inflammation is high in patients with common severe pneumonia [47], there are currently no specific guidelines for nutrition support. However, pneumonia in Covid-19 patients is usually long in duration and severe, and it is thus expected that EN should limit intestinal damage, stimulate the innate immune response and therefore help limit lung damage, while preserving muscle function, in particular respiratory function.

\section{Practical challenges for clinical nutrition during the Covid-19 epidemic}

In an epidemic context, implementation of international guidelines on nutritional screening and care may appear difficult. This is especially the case for enteral nutrition (EN) as a first-line nutrition support. Numerous French hospitals had to face staff reorganization and sometimes material shortage; in these conditions, clinical nutrition practice should adapt and sometimes be implemented as degraded procedures. Based on the experience from the first weeks of the epidemic in France, we propose here to emphasize ten challenges for clinical nutrition practice in Covid-19 patients:

1. Work modalities of clinical nutrition professionals need to be reorganized.

2. The nutrition protocols for Covid-19 patients need to be simple and easily applicable.

3. Screening for malnutrition should be feasible.

4. Energy and protein needs assessment needs to be simple and easily applicable.

5. Hospital staff should be aware of refeeding syndrome prevention.

6. Adaptation of hospital food delivery needs to be considered to overcome insufficient food intake.

7. Misconceptions should be fought, as they are challenging the use of EN.

8. Clinical situations and organizational constraints that may make EN unfeasible need to be known. 
Fig. 1 Nutrition protocol for the Covid-19 hospitalized patient under oxygen $\geq 3 \mathrm{l} / \mathrm{min}$ (adapted from sheet 8-annex -www.sfenm.org). BMI body mass index, BW body weight, $\mathrm{CVC}$ central venous catheter, EN enteral nutrition, ICU intensive care unit, IV tube, PN parenteral nutrition, PVC peripheral venous catheter. intravenous, NGT nasogastric

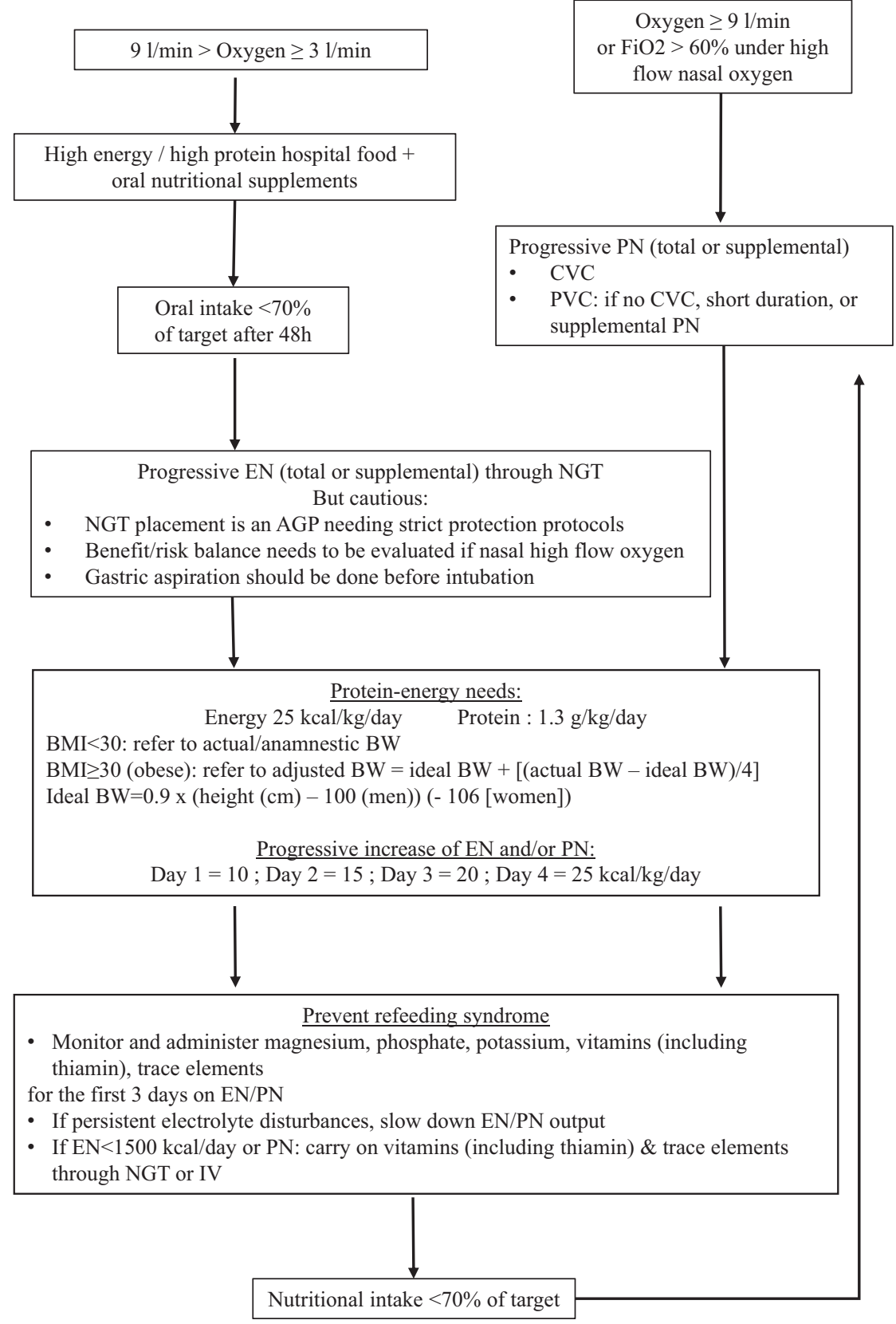

9. Parenteral nutrition (PN) may be considered as an alternative to EN.

10. Nutritional care could find a place in post-acute care rehabilitation.

Facing these challenges and barriers, our objective is to propose practical solutions for the clinical nutrition caregivers to promote nutritional care in the hospitalized Covid-19 patient. We also propose a flow chart (Fig. 1) for optimizing the nutritional management of the Covid-19 patients in the non-ICU wards, including the possible use of $\mathrm{PN}$ in the situations where the use of EN as the first line of nutrition support is considered as risky or unfeasible.

\section{Challenge 1: work modalities of clinical nutrition professionals need to be reorganized}

In the context of the Covid-19 epidemic, administrative and political rules are set up to prevent virus propagation among patients and staff. Dedicated teams of healthcare professionals are constituted including physicians, nurses, nurse assistants...chosen according to their expertise in urgent respiratory care and artificial ventilation. In that context, nutritional screening and care is not considered as a priority. The access to the Covid-19 hospital wards is restricted to these 
indispensable and dedicated healthcare professionals to prevent any propagation of the virus.

Therefore, unfortunately, in most hospitals, the dietitians and the nutrition support team are not considered indispensable. Also with the objective of preventing virus propagation, the clinical visits with non-Covid-19 hospitalized patients are sometimes restrained as well. As there is almost no way to overcome these administrative rules, clinical nutrition professionals need to reorganize their daily work. In this situation, there is a room for intra-hospital teleconsultations (e.g., dietician is calling the patient through her/his room phone, if clinically able to speak). Phone contacts remain to be kept with nurses and doctors by giving the advices for nutritional management by email or phone. To improve nutritional care, clinical nutrition teams should be involved in the writing and the implementation of clinical nutrition protocols for Covid-19 and non-Covid-19 patients. Institutional support of the importance of nutritional support should be sought.

\section{Challenge 2: the nutrition protocols for Covid-19 patients need to be simple and easily applicable}

In the French regions most hit by the epidemic, the massive hospital admissions fluxes have made it necessary to commandeer healthcare professionals not familiar with infectious diseases and internal medicine (e.g., surgeons, anaesthesiologists, nontrained nursing staff...). These professionals are also mostly not well aware of clinical nutrition procedures and protocols, such as insertion of nasogastric tube (NGT), modalities of prescriptions of enteral or PN. To reduce the risk of virus transmission, the care organization is organized so that a minimum of healthcare givers can intervene at patient bed, as personal protective equipment availability is scarce. They are asked to plan the different cares in one single visit. In that context, combined with the fact that clinical nutrition professionals (challenge 1) are not authorized to assist caregivers in the Covid-19 units, there is obviously no or little room for personalized nutritional care, as it should be. It is therefore highly needed that the nutrition protocols for Covid-19 patients are simple and easily applicable. The use of systematic procedure, instead of too complicated decision algorithms, would be preferable for healthcare professionals not familiar with clinical nutrition.

\section{Challenge 3: screening for malnutrition should be feasible}

Based on the above rationale, malnutrition should be actively investigated in the Covid-19 patient. However, the recommend full procedure for malnutrition diagnosis appears to be not fully applicable in the context of the Covid-19 epidemic: lack of scales in Covid-19 units, risk of viral transmission secondary to the use of scales, bioimpedance analyser, handgrip dynamometer, and usual tools for arm or calf circumference measurement [5]. At best, the phenotypic criteria of the Global Leadership Initiative on Malnutrition [19] and the French Health High Authority (https://www.has-sante.fr/jcms/p_3118872/fr/dia gnostic-de-la-denutrition-de-l-enfant-et-del-adulte) could be theoretically collected based on patients' or relatives' interview indicating usual weight and most recent height. However, efforts should be made to measure as soon as possible after admission the actual weight, in order to calculate a recent weight loss and a BMI. If no data on weight are available, visual inspection of the physical condition of the patient may be enough to diagnose malnutrition in the presence of severe muscle wasting. On the opposite, etiologic criteria are easily identified: (i) Covid-19, as an "acute inflammatory disease"; (ii) food intake within the past week that could be easily assessed with semiquantitative methods: an analog scale between 0 and 10/10) [48] or consumed portions $(0,1 / 4,1 / 2 ; 1)$ during lunch or dinner, as collected in the NutritionDay survey [49]; consuming $<7 / 10$ should alert to likely malnutrition $[48,50]$. So, screening should not aim to collect all possible indicators but rely on one of few criteria which are enough to identify patients already malnourished or at high-risk of becoming malnourished who constitute the target for early intervention.

\section{Challenge 4-energy and protein needs assessment needs to be simple and easily applicable}

Indirect calorimetry is the reference method to assess the energy requirements [10]. In the context of the Covid-19 epidemic, indirect calorimetry using a canopy is generally contraindicated because the usual decontamination procedures cannot be guaranteed in most wards in an epidemic context [6]. Only in some specialized ICUs, already trained for indirect calorimetry and adequately staffed, could the use of indirect calorimetry be considered for some metabolically unstable patients, e.g., staying more than 10 days in the ICU or those on full PN [6]. In that context, IC devices and virus filters only dedicated for Covid-19 patients only should be used. We recently stated [6]: "To avoid exposure to aerosol and potential virus contamination during IC device connection/disconnection, our recommendations are, previous to connection to the IC device, to put the ventilator on Stand By and to clamp the tube; then connect and when connected to declamp the tube and to restart the ventilator. This way is preventing the potential spread of virus during disconnection/connection." Even if these technical aspects of indirect calorimetry take time, we 
believe that, as indirect calorimetry should be targeted on specific patients, they could be implemented.

To determine calorie and protein needs, we therefore propose the use of predictive equations according to weight (Fig. 1). For obese patients (BMI $\geq 30$ ), adjusted body weight based on the patient's height and ideal weight should be used: adjusted weight $=$ ideal weight $+[$ (actual weight - ideal weight)/4]; ideal weight $=0.9 \times($ height $(\mathrm{cm})-100$ (male)) ( -106 [female]). For all other patients, it should be the actual body weight, which again underlines the need of measuring patient's weight, as soon as possible after admission. The ultimate goal is to avoid underfeeding or overfeeding. Patients on EN are more exposed to underfeeding because of digestive symptoms and feeding interruptions, while overfeeding may happen during PN.

\section{Challenge 5: hospital staff should be aware of refeeding syndrome prevention}

The prevention of refeeding syndrome must be systematic [51]. The Covid-19 patient is likely to be malnourished, especially after several days/weeks of evolution. The medical and nursing staff in charge of the Covid-19 patients is often unaware of the deleterious consequences of nonadapted nutrition support, especially the refeeding syndrome. Therefore, every specialized unit receiving Covid19 patients at the acute phase should have protocols available for the prevention of refeeding syndrome. It includes appropriate supplementation with electrolytes, including magnesium, potassium and phosphorus, vitamins, trace elements and close clinical and biological monitoring: monitoring of energy and protein intakes, weight (if feasible), and electrolytes, including magnesium, potassium and phosphorus [51]. The risk of refeeding syndrome is maximal under the use of PN. A Covid-19 patient's nutrition protocol is proposed in Fig. 1.

\section{Challenge 6: adaptation of hospital food delivery needs to be considered to overcome insufficient food intake}

The organization of hospital food delivery has been modified during the Covid-19 epidemic so that the meal trays are not entering into the patient rooms. This means that food intake monitoring after meal tray retrieval is impossible. Moreover, dieticians have very limited direct access to the patients. Therefore, daily adaptation of food supply to patient's intake is impossible. At best, hospital food intake should be monitored based on patient's interview (as described for screening in challenge 3 ) but it will not be possible for every patient. Therefore, to strengthen the chance of reaching the energy and protein targets (Fig. 1), we propose that every Covid-19 patient should systematically receive, from the day of hospital admission, enriched food (higher energy and protein) as available and two oral nutritional supplements each day. This is a simple and systematic protocol in response to Challenge 3. Insufficient food intake is expected to be very frequent in Covid-19 patients: anorexia, dyspnea, post-ICU dysphagia, rapid catabolism and muscular weakness, insufficient human resources to help with the meal and constraints related to isolation. In case food intake would remain insufficient after $48 \mathrm{~h}(\leq 50 \%$ of consumed food or supplements) [49] or analog scale $<7$ / $10[48,50])$, we propose that nutrition support should be indicated with EN as first line (Fig. 1).

As recommended (https://www.nutritioncare.org/uploa dedFiles/Documents/Guidelines_and_Clinical_Resources/ Nutrition\%20Therapy\%20COVID-19_SCCM-ASPEN.pdf) $[4,5,12,25,52]$, EN through a NGT should be preferred as a first line nutrition support because of its expected benefits in the Covid-19 patient (see 1st part). However, Covid-19 patients are mostly managed by medical and nonmedical staff often not familiar with nutrition support techniques. In our French experience, several objections rose against the use of EN by NGT in the context of the Covid-19 epidemic. These objections are mostly based on wrong opinions (challenge 7), while others are based on actual risks of EN in some clinical situations where usual conditions may not be guaranteed (challenge 8 ).

\section{Challenge 7-misconceptions should be fought, as they are challenging the use of EN}

\section{Misconception 1-continuous feeding increases the risks of EN intolerance}

The advantage of continuous EN administration (e.g., $50 \mathrm{ml} /$ h) is to maintain a low gastric volume, contrary to a meal or after an oral nutritional supplement of $200 \mathrm{ml}$ ingested over a few minutes [47]. Continuous feeding may also have a metabolic beneficial effect, since the VCO2 induced by nutrient oxidation is much more stable and regular by continuous EN than with intermittent meals. Finally, continuous EN is associated with better glycaemic control and less insulin resistance at the acute phase of illness [12].

\section{Misconception 2-the nasogastric tube (NGT) insertion could induce/worsen hypoxemia and apnea}

During NGT insertion procedure, the apnea is no longer than that during swallowing and the patient continues to 
breathe normally. The total apnea time is much shorter during EN infusion than during a normal meal. In other types of dyspneic patients, such as those with amyotrophic lateral sclerosis and a vital capacity lower than $30 \%$ of the theoretical, with or without noninvasive ventilation, NGT insertion was perfectly possible, safe and not different from EN through a gastrostomy in terms of day 30 complications [53].

\section{Misconception 3-NGT for EN, once in place, hinders natural nasal ventilation or the fitting of oxygen mask}

The use of a fine gauge NGT (CH8 or CH10) is recommended. This size hardly represents more than $1 / 8$ th to $1 /$ 6 th of the diameter of the nasal ostium, and does not interfere with natural breathing. Therefore, oxygen administration is compatible with NGT for EN at the same time. EN through NGT is a common practice for many malnourished patients, even elderly or at home, with respiratory failure: neuromuscular patients, chronic obstructive pulmonary disease stage IV, cystic fibrosis before lung transplantation, long-term oxygen therapy... without any risk of pneumonia or loss of effectiveness of noninvasive ventilation. Adaptation "tips" for Covid-19 patients having oxygen mask and a NGT for EN at the same time are proposed.

\section{Challenge 8-clinical situations and organizational constraints that may make EN unfeasible need to be known}

There are obvious clinical situations and organizational constraints increasing the risk of EN complications and the constraints related to NGT and EN are probably higher in Covid-19 patients than in standard medical patients:

\section{Issue 1-despite the absence of data in Covid-19 patients, the risk of EN complications may be higher in patients requiring high outflow oxygen}

Many patients are still receiving high flow nasal cannula therapy or noninvasive ventilation in many centers $[54,55]$. From a practical point of view, based on the Chinese experience, these patients are almost not fed orally or enterally. Also, as reported for standard medical patients, older and confused patients, and those with dementia [11] should be considered at increased risk of EN-related aspiration pneumonia. In these situations, if oral intake is insufficient, PN may be indicated earlier than usual, skipping the EN step (see challenge 9) (Fig. 1).
Issue 2-NGT insertion is associated with a high risk of viral contamination

Like all contact gestures potentially associated with contaminating aerosol, the healthcare professional inserting NGT for EN must wear strict antiviral protections. However, at the start of the epidemic, mask shortage in some hospital increased the risk of nurse contamination during NGT insertion.

\section{Issue 3-once NGT is inserted, chest X-ray may not always be feasible}

Some French centers have decided to perform thoracic CT as a systematic assessment of lung injury in the hospitalized Covid-19 patient in dedicated radiology rooms, and chest $\mathrm{X}$-ray has been abandoned. Therefore, bedside chest X-ray control after NGT insertion is more rarely performed, in order to reduce the risk of virus dissemination. Thus, NGT should be ideally inserted before performing the CT to take opportunity of CT to check the good location, but this is likely a rare situation.

Issue 4-in the event of a shortage of pumps with flow regulator, it is necessary to reserve them as a priority for the ICUs

EN should be performed using a pump with flow regulator. In non-intubated patients, it seems preferable not to use EN rather than doing it without pumps with flow regulator, because of the risk of aspiration pneumonia. Indeed, underfeeding is likely to have less severe consequences than aspiration pneumonia. In that context, PN through a peripheral or a central venous line could be indicated (Fig. 1). Fortunately, most French hospitals have been quickly reequipped with EN portable pumps so that a shortage is unlikely to happen.

\section{Issue 5-EN exposes to an increased risk of aspiration in case of emergency intubation}

There is no data available regarding this issue. High gastric residual volume has not been shown to be a risk factor for aspiration per se [56]. The risk of aspiration from a nonfasted stomach is probably less with a low output EN than after a full meal. To limit the risk, we advise stomach aspiration with NGT before any emergency intubation.

Facing these issues limiting the feasibility of EN, the French hospitals harder hit by the epidemic, because of work overload and material shortage, were deemed to use degraded procedures. One of them was not feeding the patient or using PN, instead of EN, to avoid NGT insertion and the risk of staff contamination. 


\section{Challenge 9-PN may be considered as an alternative to EN}

PN is usually indicated whenever EN is impossible or contraindicated, or in addition to EN as long as it is insufficient (supplemental PN) or not tolerated [12]. Beside these usual $\mathrm{PN}$ indications, all the issues reported in challenges 7 and 8 may lead to consider PN instead of EN for the Covid-19 patient. In any situation, PN should be prescribed using a case by case decision making, putting in balance the malnutrition risk and potential PN side effects (e.g., overfeeding, hyperglycemia over $10 \mathrm{mmol} / \mathrm{l}$, catheter infections, lymphangitis, refeeding syndrome...) that should be prevented [12, 57]. Pumps with flow regulator are also needed for PN infusion. If a central venous catheter, most often a peripherally inserted central catheter, can be inserted in good safety conditions, central PN should be preferred. Instead, peripheral PN could be a short-term alternative, but with a maximal duration of 10 days because of its low nitrogen content, poor venous tolerance and increased nurse workload.

\section{Challenge 10-nutritional care could find a place in post-acute care rehabilitation}

At the acute phase of the Covid-19, early optimized nutritional care and physical activity are difficult to implement. Many patients may suffer from post-ICU weakness syndrome and severe muscle loss even in non-ICU patients is common. Therefore, the post-acute care rehabilitation is warranted to enhance muscle mass and function, and recovery of the Covid-19 patients. It should combine nutritional care and physical activity. Clinical nutrition professionals should be among the key actors of this rehabilitation. As nutritional evaluation may have been limited in the acute stage, a more thorough nutritional screening should take place before discharge or on admission to the rehabilitation unit. Nutrition support may be then more easily performed in accordance with the international guidelines $[4,5]$. As the risk of interhuman or contact virus transmission even after a long hospital stay are still unknown, all the procedures should be adapted to the risk of virus transmission.

\section{Conclusion}

The Covid-19 epidemic mobilizes all the available hospital resources for an optimized care of the patients, including the most advanced ventilation techniques. Nutritional care is a key component of a global care but its implementation may be overlooked or made difficult by the epidemic context. Facing this new disease, our practice in clinical nutrition should therefore adapt, identify different care modalities, and unfortunately, rely on degraded procedures. Despite these limitations, efforts should be developed to provide some oral nutrition support at the early stage and scale up to EN or PN as needed [5, 58]. Practical fact sheets have been published by the French-speaking Clinical Nutrition and Metabolism Society (www.sfncm.org) to help clinicians to implement nutritional strategy at the different steps of Covid-19 patients' course, at hospital, during rehabilitation and back home.

Although a lot of effort is targeted on antiviral therapy, dedicated trials should evaluate whether early more intensive nutritional intervention could improve the clinical outcome of the Covid-19 patients. Dedicated studies about the nutrition status of the Covid-19 patients are now awaited to enrich our knowledge about the metabolism alterations induced by this new disease and adapt the nutrition support strategy.

Acknowledgements All the Société Francophone de Nutrition Clinique et Métabolisme (SFNCM) (www.sfncm.org) members.

Author contributions RT, MC, FJ, SMS, JB, and PD drafted the article.

\section{Compliance with ethical standards}

Conflict of interest RT received consulting and conference fees: Aguettant, Baxter, B. Braun, Fresenius-Kabi, Nutricia, Roche; conference fees: Astra-Zeneca, Homeperf, Lactalis, Nestlé, Shire; designed and received royalties for the Simple Evaluation of Food Intake ${ }^{\circledast}\left(\mathrm{SEFI}^{\circ}\right)$ (Knoë, le Kremlin Bicêtre, France). MC declared no conflict of interest. FJ received consulting and conference fees: Baxter, Fresenius-Kabi. JB received consulting and conference fees: Baxter, Fresenius-Kabi, Nestlé Health Science. SMS received consulting, conference, and investigator honoraria from B. Braun, Baxter, Cardinal Health, Fresenius-Kabi, Homeperf, Laboratoires Grand Fontaine, Nestlé Health Science, Nutricia, Theradial, Pierre Déchelotte stakeholder and consulting fees: TagEDys SA; conference fees: Baxter, Fresenius, Nestlé Health Science; consulting fees: Nestlé, Sanofi, Servier.

Publisher's note Springer Nature remains neutral with regard to jurisdictional claims in published maps and institutional affiliations.

\section{References}

1. Zhu N, Zhang D, Wang W, Li X, Yang B, Song J, et al. A novel coronavirus from patients with pneumonia in China, 2019. N Engl J Med. 2020;382:727-33.

2. Huang C, Wang Y, Li X, Ren L, Zhao J, Hu Y, et al. Clinical features of patients infected with 2019 novel coronavirus in Wuhan, China. Lancet. 2020;395:497-506.

3. Li T, Zhang Y, Gong C, Wang J, Liu B, Shi L, et al. Prevalence of malnutrition and analysis of related factors in elderly patients with COVID-19 in Wuhan, China. Eur J Clin Nutr. 2020. https://doi. org/10.1038/s41430-020-0642-3.

4. Barazzoni R, Bischoff SC, Breda J, Wickramasinghe K, Krznaric Z, Nitzan D, et al. ESPEN expert statements and practical 
guidance for nutritional management of individuals with SARSCoV-2 infection. Clin Nutr. 2020. https://doi.org/10.1016/j.clnu. 2020.03.022. pii: S0261-5614(20)30140-0.

5. Thibault R, Quilliot D, Seguin P, Tamion F, Schneider S, Déchelotte P. Nutritional care at hospital during the Covid-19 viral epidemic: expert opinion from the French-speaking Society for Clinical Nutrition and Metabolism (SFNCM). Nutr Clin Metab. 2020;34:97-104.

6. Thibault R, Seguin P, Tamion F, Pichard C, Singer P. Nutrition of the COVID-19 patient in the intensive care unit (ICU): a practical guidance. Crit Care. 2020;24:447. https://doi.org/10.1186/s13054020-03159-z.

7. Caccialanza R, Laviano A, Lobascio F, Montagna E, Bruno R, Ludovisi $\mathrm{S}$, et al. Early nutritional supplementation in noncritically ill patients hospitalized for the 2019 novel coronavirus disease (COVID-19): Rationale and feasibility of a shared pragmatic protocol. Nutrition. 2020;74:110835.

8. Simonnet A, Chetboun M, Poissy J, Raverdy V, Noulette J, Duhamel A, et al. High prevalence of obesity in severe acute respiratory syndrome coronavirus-2 (SARS-CoV-2) requiring invasive mechanical ventilation. Obesity. 2020. https://doi.org/10. 1002/oby.22831.

9. Ong SWX, Young BE, Leo YS, Lye DC. Association of higher body mass index (BMI) with severe coronavirus disease 2019 (COVID-19) in younger patients. Clin Infect Dis. 2020. https:// doi.org/10.1093/cid/ciaa548. pii: ciaa548m.

10. Fang L, Karakiulakis G, Roth M. Are patients with hypertension and diabetes mellitus at increased risk for COVID-19 infection? Lancet Respir Med. 2020. https://doi.org/10.1016/S2213-2600 (20)30116-8.

11. Volkert D, Beck AM, Cederholm T, Cruz-Jentoft A, Goisser S, Hooper L, et al. ESPEN guideline on clinical nutrition and hydration in geriatrics. Clin Nutr. 2019;38:10-47.

12. Singer P, Blaser AR, Berger MM, Alhazzani W, Calder PC, Casaer MP, et al. ESPEN guideline on clinical nutrition in the intensive care unit. Clin Nutr. 2019;38:48-79.

13. Lüers JC, Klußmann JP, Guntinas-Lichius O. The COVID-19 pandemic and otolaryngology: what it comes down to? Laryngorhinootologie. 2020;99:287-91.

14. Xiao F, Tang M, Zheng X, Li X, Shan H. Evidence for gastrointestinal infection of SARS-CoV-2. Gastroenterology. 2020;158:1831-3.

15. Cheung KS, Hung IF, Chan PP, Lung KC, Tso E, Liu R. Gastrointestinal manifestations of SARS-CoV-2 infection and virus load in fecal samples from the Hong Kong Cohort and systematic review and meta-analysis. Gastroenterology. 2020. https://doi.org/ 10.1053/j.gastro.2020.03.065. pii: S0016-5085(20)30448-0.

16. Yeo C, Kaushal S, Yeo D. Enteric involvement of coronaviruses: is faecal-oral transmission of SARS-CoV-2 possible? Lancet Gastroenterol Hepatol. 2020;5:335-7.

17. Gu J, Han B, Wang J. COVID-19: gastrointestinal manifestations and potential fecal-oral transmission. Gastroenterology. 2020;158:1518-9.

18. Jeevanandam M, Young DH, Schiller WR. Obesity and the metabolic response to severe multiple trauma in man. J Clin Investig. 1991;87:262-9.

19. Cederholm T, Jensen GL, Correia MITD, Gonzalez MC, Fukushima R, Higashiguchi T, et al. GLIM criteria for the diagnosis of malnutrition-a consensus report from the global clinical nutrition community. Clin Nutr. 2019;38:1-9.

20. Huang R, Zhu L, Xue L, Liu L, Yan X, Wang J, et al. Clinical findings of patients with coronavirus disease 2019 in Jiangsu province, China: a retrospective, multi-center study. PLoS Negl Trop Dis. 2020;14:e0008280. https://doi.org/10.1371/journal. pntd.0008280.

21. Richardson S, Hirsch JS, Narasimhan M, Crawford JM, McGinn T, Davidson KW, et al. Presenting characteristics, comorbidities, and outcomes among 5700 patients hospitalized with COVID-19 in the New York City area. JAMA. 2020;323:2052-9.

22. Zhou F, Yu T, Du R, Fan G, Liu Y, Liu Z, et al. Clinical course and risk factors for mortality of adult inpatients with COVID-19 in Wuhan, China: a retrospective cohort study. Lancet. 2020;395: 1054-62.

23. Schuetz P, Fehr R, Baechli V, Geiser M, Deiss M, Gomes F, et al. Individualised nutritional support in medical inpatients at nutritional risk: a randomised clinical trial. Lancet. 2019;393:2312-21.

24. Liu Y, Yang Y, Zhang C, Huang F, Wang F, Yuan J, et al. Clinical and biochemical indexes from 2019-nCoV infected patients linked to viral loads and lung injury. Sci China Life Sci. 2020;63:364-74.

25. Chapple LS, Fetterplace K, Asrani V, Burrell A, Cheng AC, Collins $\mathrm{P}$, et al. Nutrition management for critically and acutely unwell hospitalised patients with coronavirus disease 2019 (COVID-19) in Australia and New Zealand. Aust Crit Care. 2020;33:399-406.

26. Zheng HY, Zhang M, Yang CX, Zhang N, Wang XC, Yang XP, et al. Elevated exhaustion levels and reduced functional diversity of $\mathrm{T}$ cells in peripheral blood may predict severe progression in COVID-19 patients. Cell Mol Immunol. 2020;17:541-3.

27. Ardawi MS. Glutamine and glucose metabolism in human peripheral lymphocytes. Metabolism. 1988;37:99-103.

28. Carr EL, Kelman A, Wu GS, Gopaul R, Senkevitch E, Aghvanyan A, et al. Glutamine uptake and metabolism are coordinately regulated by ERK/MAPK during $\mathrm{T}$ lymphocyte activation. J Immunol. 2010;185:1037-44.

29. Manhart N, Vierlinger K, Spittler A, Bergmeister H, Sautner T, Roth E. Oral feeding with glutamine prevents lymphocyte and glutathione depletion of Peyer's patches in endotoxemic mice. Ann Surg. 2001;234:92-7.

30. Savoye G, Miralles-Barrachina O, Déchelotte P, Belmonte-Zalar L, Brung-Lefebvre M, Zalar A, et al. Low levels of gastric mucosal glutathione during upper gastric bleeding associated with the use of nonsteroidal anti-inflammatory drugs. Eur J Gastroenterol Hepatol. 2001;13:1309-13.

31. Miralles-Barrachina O, Savoye G, Belmonte-Zalar L, Hochain P, Ducrotté P, Hecketsweiler B, et al. Low levels of glutathione in endoscopic biopsies of patients with Crohn's colitis: the role of malnutrition. Clin Nutr. 1999;18:313-7.

32. Bañuls C, de Marañon AM, Veses S, Castro-Vega I, LópezDomènech S, Salom-Vendrell $\mathrm{C}$, et al. Malnutrition impairs mitochondrial function and leukocyte activation. Nutr J. 2019;18:89.

33. Weindruch R, Lane MA, Ingram DK, Ershler WB, Roth GS. Dietary restriction in rhesus monkeys: lymphopenia and reduced mitogen-induced proliferation in peripheral blood mononuclear cells. Aging. 1997;9:304-8.

34. Contreras NA, Fontana L, Tosti V, Nikolich-Žugich J. Calorie restriction induces reversible lymphopenia and lymphoid organ atrophy due to cell redistribution. Geroscience. 2018;40:279-91.

35. Fock RA, Blatt SL, Beutler B, Pereira J, Tsujita M, de Barros FE, Borelli P. Study of lymphocyte subpopulations in bone marrow in a model of protein-energy malnutrition. Nutrition. 2010;26:1021-8.

36. Schneider S, Hébuterne X, Benzaken S, Hastier P, Tran A, Rampal P. Effects of cyclic enteral nutrition on the immunological status of malnourished patients. Clin Nutr. 1996;15:189-95.

37. Luo S, Zhang X, Xu H. Don't overlook digestive symptoms in patients with 2019 novel coronavirus disease (COVID-19). Clin Gastroenterol Hepatol. 2020. https://doi.org/10.1016/j.cgh.2020. 03.043. pii: S1542-3565(20)30401-8.

38. Jin X, Lian JS, Hu JH, Gao J, Zheng L, Zhang YM, et al. Epidemiological, clinical and virological characteristics of 74 cases of coronavirus-infected disease 2019 (COVID-19) with gastrointestinal symptoms. Gut. 2020;69:1002-9. 
39. Sencio V, Barthelemy A, Tavares LP, Machado MG, Soulard D, Cuinat $\mathrm{C}$, et al. Gut dysbiosis during influenza contributes to pulmonary pneumococcal superinfection through altered shortchain fatty acid production. Cell Rep. 2020;30:2934-47.

40. Latorre M, Krishnareddy S, Freedberg DE. Microbiome as mediator: do systemic infections start in the gut? World J Gastroenterol. 2015;21:10487-92.

41. Mukherjee S, Hanidziar D. More of the gut in the lung: how two microbiomes meet in ARDS. Yale J Biol Med. 2018;91:143-9.

42. Dickson RP, Singer BH, Newstead MW, Falkowski NR, ErbDownward JR, Standiford TJ, Huffnagle GB. Enrichment of the lung microbiome with gut bacteria in sepsis and the acute respiratory distress syndrome. Nat Microbiol. 2016;1:16113.

43. Yitbarek A, Taha-Abdelaziz K, Hodgins DC, Read L, Nagy É, Weese JS, et al. Gut microbiota-mediated protection against influenza virus subtype H9N2 in chickens is associated with modulation of the innate responses. Sci Rep. 2018;8:13189.

44. Wan X, Bi J, Gao X, Tian F, Wang X, Li N, Li J. Partial enteral nutrition preserves elements of gut barrier function, including innate immunity, intestinal alkaline phosphatase (IAP) level, and intestinal microbiota in mice. Nutrients. 2015;7:6294-312.

45. Sun H, Bi J, Lei Q, Wan X, Jiang T, Wu C, Wang X. Partial enteral nutrition increases intestinal sIgA levels in mice undergoing parenteral nutrition in a dose-dependent manner. Int J Surg. 2018;49:74-9.

46. Tian F, Heighes PT, Allingstrup MJ, Doig GS. Early enteral nutrition provided within $24 \mathrm{~h}$ of ICU admission: a meta-analysis of randomized controlled trials. Crit Care Med. 2018;46: 1049-56.

47. Dünnwald T, Gatterer H, Faulhaber M, Arvandi M, Schobersberger W. Body composition and body weight changes at different altitude levels: a systematic review and meta-analysis. Front Physiol. 2019;10:430.

48. Thibault R, Goujon N, Le Gallic E, Clairand R, Sébille V, Vibert $\mathrm{J}$, et al. Use of 10-point analogue scales to estimate dietary intake: a prospective study in patients nutritionally at-risk. Clin Nutr. 2009;28:134-40.
49. Hiesmayr M, Schindler K, Pernicka E, Schuh C, SchoenigerHekele A, Bauer P, et al. Decreased food intake is a risk factor for mortality in hospitalised patients: the NutritionDay survey 2006. Clin Nutr. 2009;28:484-91.

50. Bouëtté G, Esvan M, Appel K, Thibault R. A visual analogue scale for food intake as a screening test for malnutrition in the primary care setting: prospective non-interventional study. Clin Nutr. 2020. https://doi.org/10.1016/j.clnu.2020.04.042.

51. Berger MM, Reintam-Blaser A, Calder PC, Casaer M, Hiesmayr MJ, Mayer K, et al. Monitoring nutrition in the ICU. Clin Nutr. 2019;38:584-93.

52. Taylor BE, McClave SA, Martindale RG, Warren MM, Johnson $\mathrm{DR}$, Braunschweig $\mathrm{C}$, et al. Guidelines for the provision and assessment of nutrition support therapy in the adult critically Ill patient: Society of Critical Care Medicine (SCCM) and American Society for Parenteral and Enteral Nutrition (A.S.P.E.N.). Crit Care Med. 2016;44:390-438.

53. Banfi P, Volpato E, Valota C, D'Ascenzo S, Alunno CB, Lax A, et al. Use of noninvasive ventilation during feeding tube placement. Respir Care. 2017;62:1474-84.

54. Novel coronavirus pneumonia diagnosis and treatment plan (provisional 7th ed.). 2020/03/04. http://www.gov.cn/zhengce/zhengceku/ 2020-03/04/5486705/files/ae61004f930d47598711a0d4cbf874a9.pdf.

55. Alhazzani W, Moller MH, Arabi YM, Loeb M, Gong MN, Fan E, et al. Surviving Sepsis campaign on the management of critically ill adults with coronavirus disease 2019 (COVID-19). Intensive Care Med. 2020;46:854-87.

56. Westphal M. Science and fiction in critical care: established concepts with or without evidence? Crit Care. 2019;23:125.

57. Ziegler TR. Parenteral nutrition in the critically ill patient. N Engl J Med. 2009;361:1088-97.

58. Schneider SM, Albert V, Barbier N, Barnoud D, Bouteloup C, Chambrier $\mathrm{C}$, et al. Changes in care of home artificial nutrition patients during the COVID-19 epidemics in France: position of the French-speaking Society for Clinical Nutrition and Metabolism (SFNCM)'s Home Artificial Nutrition Committee. Nutr Clin Metab. 2020;34:105-7. 